\section{- Kann die PREFERE-Studie erfolgreich beendet werden?}

\author{
Um wichtige Fragen der Alltagsversorgung beantworten zu können, brauchen \\ wir hochwertige klinische Untersuchungen. Mit der PREFERE-Studie hofft man \\ Antworten auf offene Fragen zur Therapie des Niedrigrisiko-Prostatakarzinoms \\ zu finden. Doch diese werden die Studienverantwortlichen voraussichtlich \\ schuldig bleiben, denn nicht jedes uns berührende Problem lässt sich durch \\ einen randomisierten Trial lösen.
}

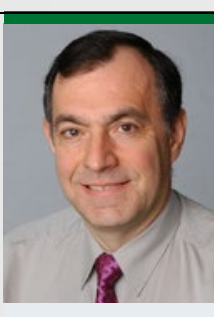

Diese Frage beantwortet für Sie

Dr. med. Reinhold Schaefer Urologie Bonn-RheinSieg

Theaterplatz 18 Praxis Bad Godesberg 53177 Bad Godesberg E-Mail: rmschaefer@telemed.de
V om Ansatz her war die PREFERE-Studie sehr sinnvoll gestaltet, um damit beim Prostatakarzinom (PCa) mit einem histologischen Gleason $3+3$ beziehungsweise $3+4$ die Wahrscheinlichkeit für die richtige Therapieakzeptanz zu ermitteln. Leider haben die Designer der Studie jedoch fatale Fehler gemacht, weil sie nicht mit den niedergelassenen Kollegen zusammengearbeitet haben, welche die Patienten primär sehen, aufklären und rekrutieren müssen. Der Chefarzt operiert die ihm zugewiesenen Patienten und schöpft hieraus seine Erfahrung. Die Nöte der definitiven Therapieentscheidung bleiben ihm erspart. Das führt zu Fehleinschätzungen bezüglich der Willensbildung und Entscheidungsfreude der Betroffenen. So erklären sich offenbar die überschätzten Zahlen der zu rekrutierenden Patienten, die von den Klinikern zugrunde gelegt wurden.

Eine Studie mit vier Therapiearmen ins Leben zu rufen, wenn zu untersuchen ist, von welcher der vier Behandlungsoptionen (radikale Prostatektomie, perkutane Strahlentherapie, Brachytherapie oder aktive Überwachung) Patienten langfristig am meisten profitieren, zeigt Unverständnis bei derartigen Therapieoptionen - zumal diese sich im primären Studienziel nicht unterscheiden. Erst nachdem die Designer die Studienvorlage fertiggestellt hatten, informierte man uns niedergelassenen Urologen. So konnten weder Änderungswünsche vorgebracht noch überhaupt etwas geändert werden. Ein Protokoll „verdeckt“ $z u$ erstellen und die Auflage, dieses geheimzuhalten auszugeben, ist ein Novum in der Wissenschaft - wie wir sie verstehen.

\section{Patienten bemängeln Lotterieverfahren}

Obwohl uns die Biometriefehler bewusst waren, haben wir niedergelassenen Urologen versucht, Männer, die mit dem Befund eines lokal begrenzten Prostatakarzinoms bei uns vorstellig oder von uns diagnostiziert wurden, zu rekrutieren. Das Dilemma um die Histologie kenne ich und will ich nicht weiter vertiefen. Nehmen wir einmal an, alle Befunde seien valide: In diesem Fall muss der Patient beraten werden und offen und ehrlich alle Optionen der Therapie genannt bekommen. Die meisten Männer fragen ihren vertrauten Arzt nach der Option, die er an ihrer Stelle wählen würde. Das ist nur allzu menschlich, auch ich habe so bei meiner Leukämie vor 13 Jahren gehandelt. Es ist nicht hilfreich, dem Patienten einen bunten Strauß an Möglichkeiten anzubieten und ihm dann zu sagen, dass in einer "tollen“ Studie andere über sein Schicksal entscheiden. Die Wahl zwischen zwei Optionen ist noch nachvollziehbar und auch vermittelbar, aber das Los zwischen reinem
Beobachten (Nichtstun), verschiedenen Bestrahlungsarten und einer Operation mit erheblichen Nebenwirkungen auf die Lebensqualität entscheiden zu lassen, nicht mehr. Das habe ich mehrfach in meiner Praxis erfahren müssen.

Studienerfahrung hat unsere Praxis reichlich. Trotz langen Gesprächen haben mehr als fünf Patienten der Teilnahme an der Studie nicht zugestimmt. Auch die Zeit, die wir uns in solchen Fällen nehmen, steht somit in keinem Verhältnis zu dem erzielbaren Erlös. So zieht man, wenn man selbst mit „Engelszungen“ keine Akzeptanz erreicht, die Konsequenz und legt die Studie ad acta. Vielen Kollegen erging und ergeht es so, die denselben Versuch wie ich unternommen haben. An der Zeit zur Aufklärung hat es mit Sicherheit nicht gelegen. Darüber hinaus weigern sich auch viele Kliniken, an der Studie teilzunehmen - warum wohl?

\section{Studienergebnisse bleiben nutzlos}

Die Veröffentlichungen in der Laienpresse haben das Urteil über die Studie wohl kaum verbessert. Die Hauptschuld niedergelassenen Urologen in die Schuhe zu schieben, war bisher das Erbärmlichste, was ich von klinischen Kollegen erfahren habe. In dramatischen Artikeln wurde vor dem Scheitern der Studie gewarnt und mit dem "guten“ Ruf der Urologen verknüpft. Ich frage mich wirklich, was schlimmer ist: zuzugeben, dass eine Studie aufgrund von Designfehlern vorzeitig beendet werden muss, also einen Fehler einzugestehen und dafür die Verantwortung zu übernehmen, oder mit der Peitsche auf die niedergelassenen Kollegen los zu gehen und ihnen die Schuld für das Dilemma zuzuschieben. Letzteres Verhalten hat sicher nicht dazu beigetragen, dass die Studie besser verstanden wird - auch wenn die Rekrutierungszahlen angeblich kurzfristig angestiegen sein sollen.

Ich frage mich ohnehin, was man mit dem Ergebnis in etlichen Jahren anfangen will, denn dann werden mehr als die Hälfte der Behandlungsmöglichkeiten nicht mehr aktuell sein, weil modernere Methoden Operationen und Bestrahlungen abgelöst haben.

Aber die Deutsche Gesellschaft für Urologie will mit aller Macht das Projekt durchboxen. Dazu scheinen ihr alle Mittel recht. Leider haben Krankenkassen und Spender mit abenteuerlichen Argumenten einer Verlängerung der Studie zugestimmt. Dies wird am Ergebnis nichts ändern: Die Studie wird scheitern. Selbst wenn am Ende ein Ergebnis erreicht wird, wird es für unsere Arbeit beim Niedrigrisiko-PCa in 30 Jahren keinen Einfluss mehr haben. 